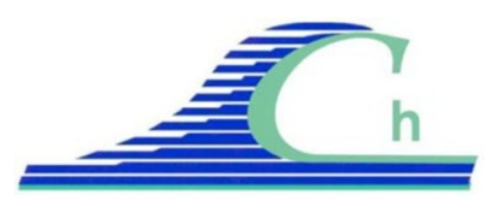

XII ${ }^{\text {èmes }}$ Journées Nationales Génie Côtier - Génie Civil

Cherbourg, 12-14 juin 2012

DOI:10.5150/jngcgc.2012.055-T @ C Editions Paralia CFL

disponible en ligne - http://www.paralia.fr - available online

\title{
MobiTC, outil de calcul automatique de l'évolution historique du trait de côte : exemples sur 3 types de morphologies côtières
}

\author{
Céline TRMAL ${ }^{1}$, Frédéric PONS ${ }^{1}$, François SABATIER ${ }^{2}$
}

1. Centre d’Étude Techniques de l’Équipement Méditerranée - CS 70499, 13593 Aix-en-Provence Cedex 3, France.

celine.trmal@developpement-durable.gouv.fr ; frederic.pons@developpement-durable.gouv.fr

2. Aix Marseille Université, Centre Européen de Recherche et d'Enseignement des Géosciences de l'Environnement, 13545 Aix-en-Provence Cedex 04, France. sabatier@cerege.fr

\section{Résumé :}

Le CETE Méditerranée a développé un outil permettant de quantifier l'évolution historique du trait de côte, avec une volonté forte de rapidité et de simplicité. Cet outil tient son originalité par rapport à des outils similaires par la génération automatique d'une ligne de base aux calculs, les statistiques étant menées trace par trace, perpendiculaire à cette ligne de base. La ligne de base passe à travers les traits de côte disponibles et donc suit la forme du littoral. Cette méthode améliore les calculs et permet de déterminer l'évolution, en une seule phase, sur un long linéaire de côte ou sur des morphologies de côte complexes comme les flèches sableuses ou des baies successives.

MobiTC, acronyme de MoBilité du Trait de Côte intègre l'état de l'art des calculs d'évolution, y compris les métadonnées comme l'incertitude sur la position des traits de côte et propose plusieurs rendus pour aider l'utilisateur à l'interprétation des résultats.

Dans cet article les potentiels de MobiTC, outil gratuit, seront illustrés par son application sur 3 sites méditerranéens de caractéristiques différentes : une longue côte sableuse, une plage de poche et une flèche sableuse.

\section{Mots-clés :}

Érosion côtière - Traits de côte - Analyse diachronique - Cartographie - Logiciel

\section{Introduction}

Aujourd'hui, en France, il existe un besoin de simplification et d'homogénéisation du traitement des traits de côte. Les politiques publiques concernant en particulier la stratégie nationale de gestion du trait de côte et la prévention des risques littoraux en sont les premières demandeuses. Pour y remédier, il est apparu nécessaire de développer un outil simple et rapide à mettre en œuvre, intégrant tout l'état de l'art du domaine. L’originalité de MobiTC par rapport au logiciel similaire comme DSAS (module 
complémentaire d'ArcGIS, HIMMELSTOSS (2009)) est la génération automatique de la ligne de base des calculs et l'intégration de nombreux indicateurs de la tendance d'évolution mais aussi des rythmes de la mobilité du trait de côte.

Il intègre les différentes positions du trait de côte mais aussi les métadonnées des traits de côte historiques. Par exemple, des informations sur les méthodes d'acquisition des levées, leurs incertitudes, sont prises en compte. Il procure également un moyen de capitaliser les traits de côte historiques.

Les rendus graphiques et les cartographies des indicateurs calculés procurent à l'utilisateur une aide à l'interprétation des nombreux résultats.

L'outil MobiTC est autonome et compatible avec le plus grand nombre de logiciel SIG de part les exports et imports notamment au format mif/mid. Les trois exemples présentés dans cet article montrent les différentes possibilités de MobiTC sur des types de côte différents.

\section{La génération automatique de la ligne de base}

Dans la littérature, une majorité des études de la mobilité du trait de côte se font à partir d'un relevé des différentes positions des traits de côte historiques sur des traces perpendiculaires à une ligne de base. La forme de la ligne de base conditionne les traces et donc les calculs d'évolution.

Cette ligne de base est en général pour ne pas dire dans tous les cas réalisée à la main et suit plus ou moins la tendance générale du trait de côte. Cette étape peut devenir fastidieuse sur de grandes étendues de plusieurs kilomètres, notamment pour des formes complexes de rivage et se révèle très subjective et non reproductible.

Une des originalités de MobiTC est de générer une ligne de base automatiquement, celle-ci suit la tendance des traits de côte. Le principe de génération est basé sur la squelettisation. Un squelette est l'objet le plus simple décrivant les lignes moyennes d'une forme quelconque (PONS, 2010). Ici, la forme est l'enveloppe contenant tous les traits de côte historiques. Cette enveloppe est obtenue par une triangularisation s'appuyant sur des points des traits de côte historiques avec un seuil en longueur des éléments. La squelettisation programmée dans MobiTC utilise les diagrammes de Voronoï. La figure 1 résume les différentes étapes de la génération de la ligne de base.

Les traces sont ensuite générées perpendiculairement à cette ligne de base et tous les calculs sont ensuite basés sur les intersections entre les traits de côte historiques et les traces.

\section{L’intégration des métadonnées dans l'outil MobiTC}

L'outil MobiTC intègre dans ces calculs les métadonnées sur les traits de côte historiques. Il est proposé à l'utilisateur de stocker les métadonnées dans un tableur. Le nom donné au fichier contenant les traits de côte pour le traitement dans MobiTC intègre les métadonnées les plus importantes. 


\section{XII ${ }^{\text {èmes }}$ Journées Nationales Génie Côtier - Génie Civil \\ Cherbourg, 12-14 juin 2012}

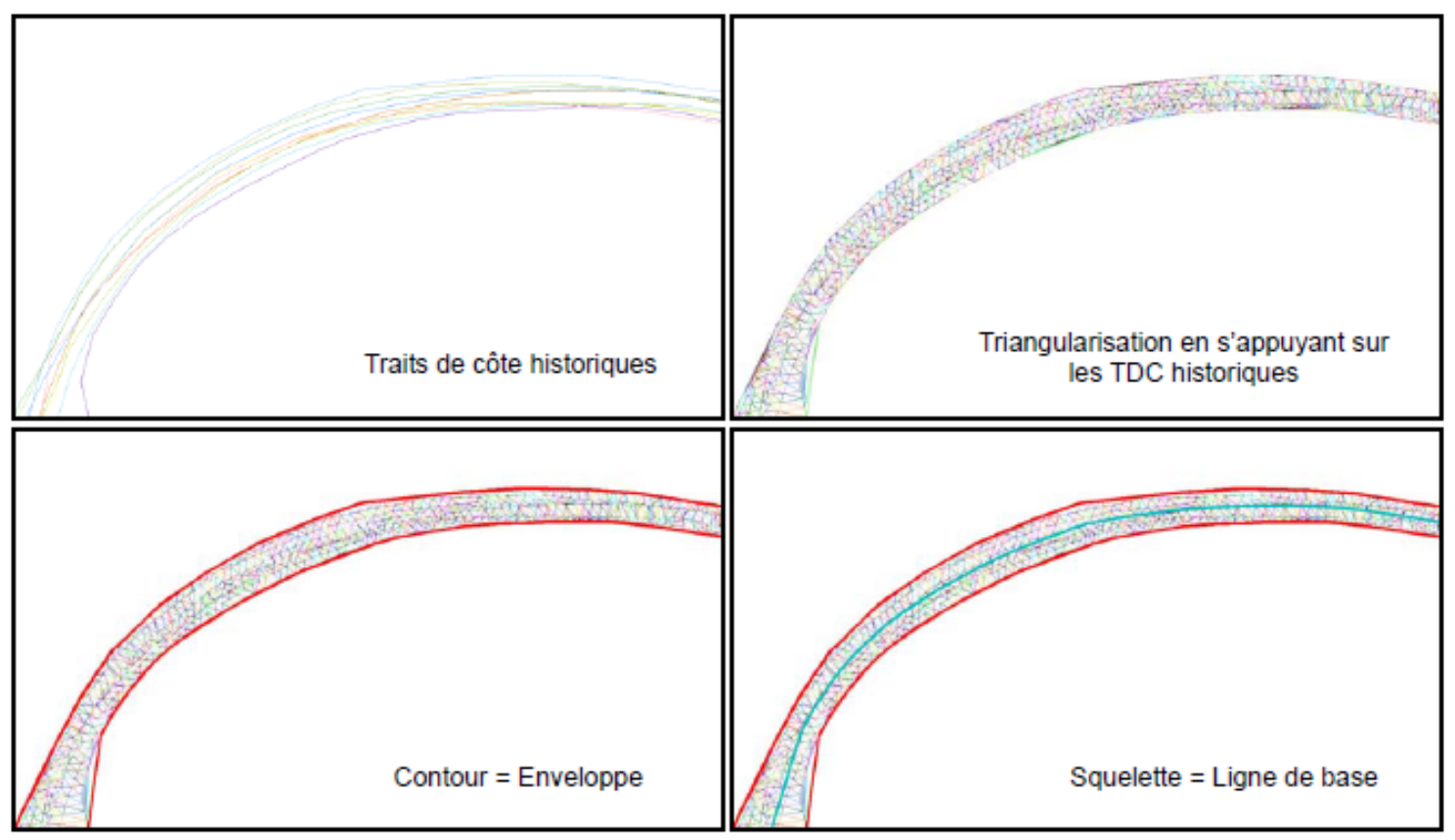

Figure 1. Étape de la squelettisation.

\subsection{Le traitement des incertitudes dans MobiTC}

Les incertitudes liées aux traits de côte peuvent être classées en deux grandes catégories :

- les incertitudes liées à la position du trait de côte, celles-ci peuvent être de plusieurs ordres: types de levés ou de numérisation, supports pour la numérisation, aux conditions climatiques, ... . Par exemple, CROWELL et al. (1991) ont estimé les différentes incertitudes qui s'additionnent lors de la digitalisation de trait de côte à partir des cartes topographiques américaines et de photographies aériennes ;

- les incertitudes liées à la date du trait de côte, ces incertitudes sont directement liées au manque de rigueur dans la conservation des métadonnées des traits de côte.

Ces incertitudes sont prises en compte dans MobiTC par tout d'abord un affichage de la barre d'erreur tant sur la position du trait de côte que sur sa date dans les graphiques des tendances linéaires trace par trace et également dans les calculs des différents paramètres de la mobilité du trait de côte.

Un exemple en est le calcul de la tendance linéaire à l'aide de la méthode des moindres carrés pondérés (WLS dans GENZ et al., 2007). L'intervention de chaque trait de côte dans le calcul de la tendance linaire est pondérée par l'inverse de l'incertitude au carré.

\subsection{L'affichage selon les métadonnées}

Les rendus des calculs effectués trace par trace prennent la forme d'un graphique représentant les positions des traits de côte en fonction des années. Sur le graphique par la forme du symbole représentant la position du trait de côte l'utilisateur peut connaître 
la nature du trait de côte (levé in situ ou numérique). La couleur du symbole donne le type de limite correspondant au trait de côte (25 types de limites ont été recensés : jet de rive, pied de falaise, ...). Les propriétaires des traits de côte sont également explicitement cités. La figure 3 en montre 2 exemples.

\section{L'aide à l'interprétation des résultats}

\subsection{De nombreux indicateurs d'évolution}

De manière à guider l'utilisateur dans l'analyse de la mobilité du trait de côte, de nombreux indicateurs pertinents recensés dans la littérature sont disponibles.

Concernant les évolutions trace par trace, GENZ et al. (2007) en fait une liste complète et critique. Actuellement MobiTC intègre le calcul de la tendance linéaire avec ces intervalles de confiance, la tendance linéaire avec une pondération des incertitudes des traits de côte, un facteur de complexité du modèle permettant de détecter des tendances non-linéaire et les changements de tendance (FENSTER \& DOLAN, 1994).

Il est également possible d'étudier les distributions de chaque paramètre (par ex. tendance linéaire) par ligne de base. Le calcul du spectre permet également de déterminer les formes cycliques du littoral ainsi que leur longueur d'onde (WALTON 1999).

L'objectif à terme est d'en intégrer un maximum dans MobiTC tout en guidant l'utilisateur dans le jugement de la pertinence de leur calcul au cas par cas.

\subsection{Des visualisations SIG simultanées}

Un soin particulier a été donné aux rendus et sorties de l'outil MobiTC. L'objectif est d'éviter au maximum les opérations fastidieuses de mise en forme des résultats par les utilisateurs familiers ou non avec les SIG.

Il est tout d'abord possible de visualiser les différents paramètres en même temps avec par défaut une accentuation des problèmes. Par exemple sur la figure 4 et la figure 5 , un coefficient de régression proche de 0 apparaît en noir, la tendance n'est pas valable. De même un nombre de traits de côte faible apparaît en orange foncé.

Il est également possible de traiter simultanément plusieurs secteurs. Le calcul de la ligne de base se fait à partir de tous les traits de côtes des différents secteurs. Ainsi autant de lignes de base se créent que de secteurs. Ceci permet au final d'avoir les mêmes échelles de couleur pour tous les secteurs. L'utilisateur peut ainsi évaluer, comparer un secteur par rapport à un autre et les hiérarchiser.

\section{Trois exemples d'application de morphologies côtières différentes}

Lors de son développement l'outil MobiTC a été testé sur plusieurs configurations de côte de manière à s'adapter à toutes les géométries de côte. L'objectif est toujours de limiter au maximum les manipulations de fichiers ou de logiciels. Ci-dessous trois 


\section{XII ${ }^{\text {èmes }}$ Journées Nationales Génie Côtier - Génie Civil \\ Cherbourg, 12-14 juin 2012}

exemples de configuration de côte sableuse sont présentés. Il est à noter que l'outil a également été testé avec succès sur des côtes à falaises en Manche.

Les résultats (taux d'évolution, ...) de ces 3 exemples ne sont pas à regarder en tant que tels, ces secteurs ont été étudiés pour la validation de MobiTC, les résultats peuvent être imprécis du fait que certains traits de côte ne contenaient pas suffisamment de métadonnées (date imprécise, mode d'acquisition et précision inconnus, ...).

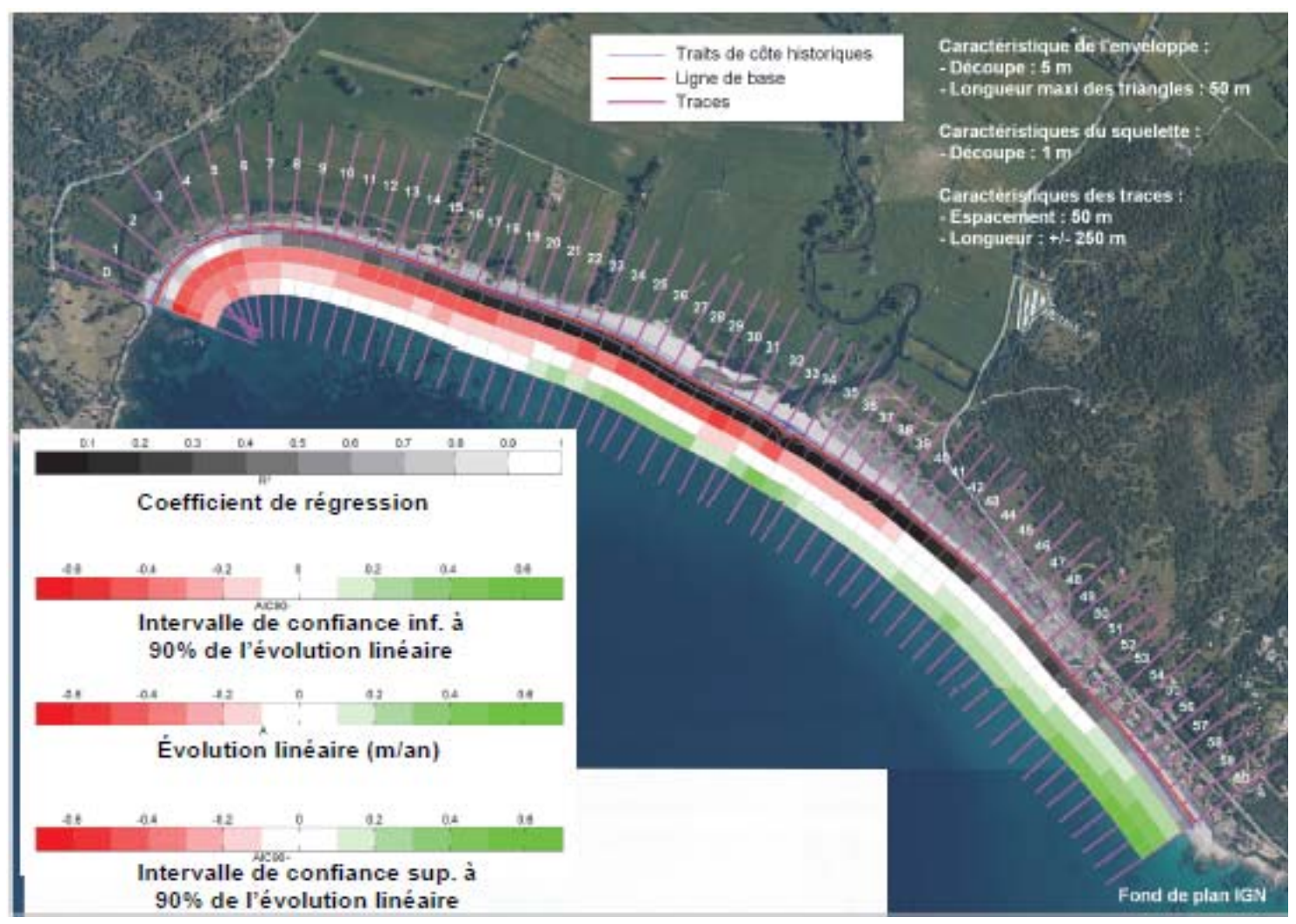

Figure 2. Traitement d'une plage de poche par MobiTC.

\subsection{Test 1 : Une plage de poche}

Sur cette plage de poche (figures 2 et 3), une dizaine de traits de côte ont été digitalisés à partir de photos aériennes ou de l'Orthophoto de l'IGN. Cet exemple illustre le fait que la ligne de base générée de façon automatique suit la tendance des traits de côte. 

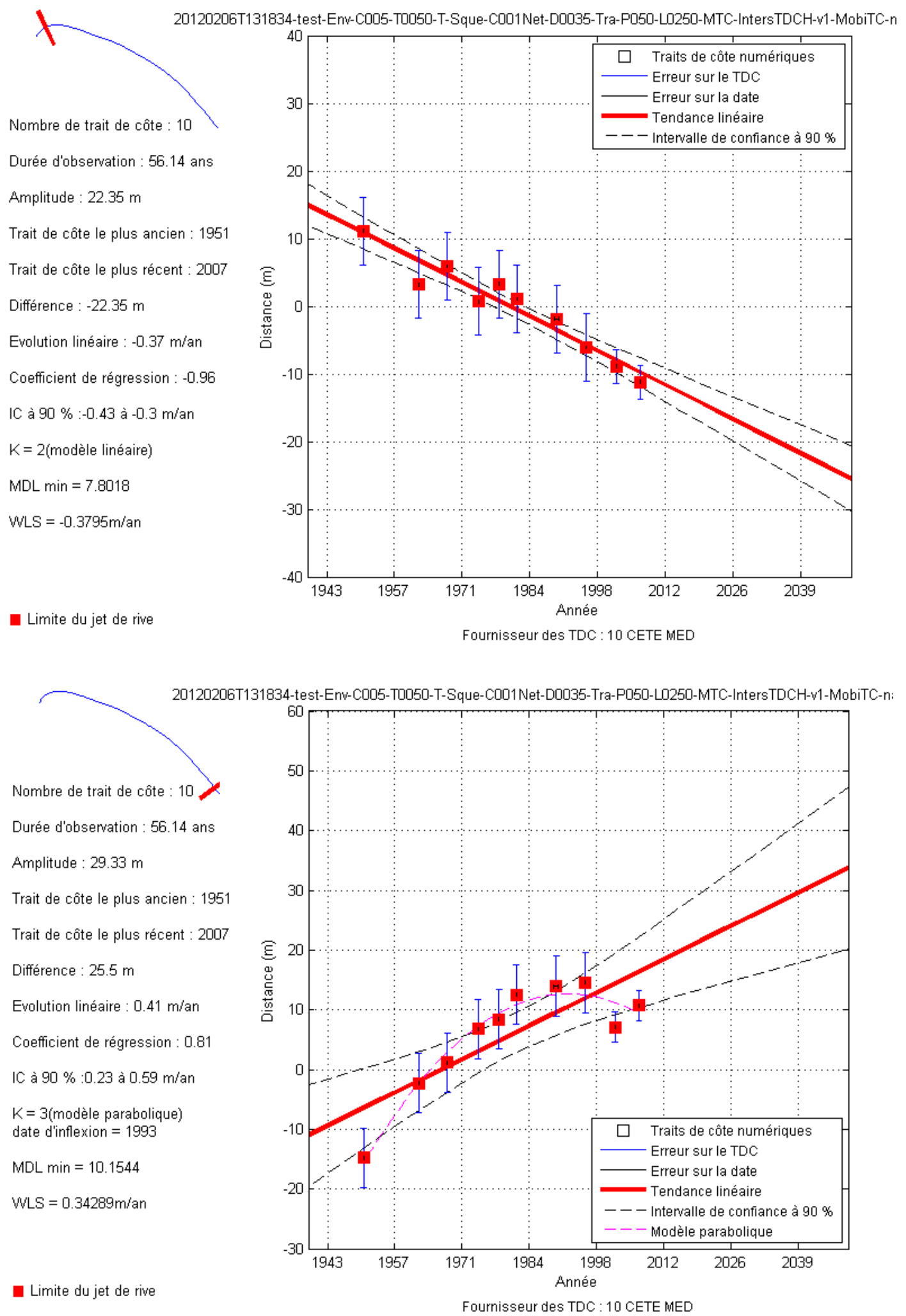

Figure 3. Exemple de graphique sur 2 traces ( $n^{\circ} 4$ et 58 de la figure 2). 


\section{XII ${ }^{\text {èmes }}$ Journées Nationales Génie Côtier - Génie Civil \\ Cherbourg, 12-14 juin 2012}

\subsection{Test 2 : Une grande étendue sableuse}

Dans cet exemple, toute la Camargue (ligne de base de $88 \mathrm{~km}$ ) a été traitée d'un seul tenant. 16 traits de côte historiques ont été utilisés. Ils n'ont pas été retravaillés de façon à nettoyer les estuaires, ce qui améliorerait la ligne de base. D'ailleurs pour la réalisation de la ligne de base, les années 1823, 1841 et 1895 n'ont pas été sélectionnées car les traits de côte contenaient de nombreuses îles au niveau du delta du Rhône mais utilisées dans les tendances.

La figure 4 représente la ligne de base et les résultats, par soucis d'affichage les traces sont espacées d'un kilomètre. Les résultats sélectionnés sont le nombre de date en dégradé d'orange, le coefficient de régression de l'ajustement linéaire en dégradé de noir et le taux linéaire d'évolution en dégradé de rouge à vert.

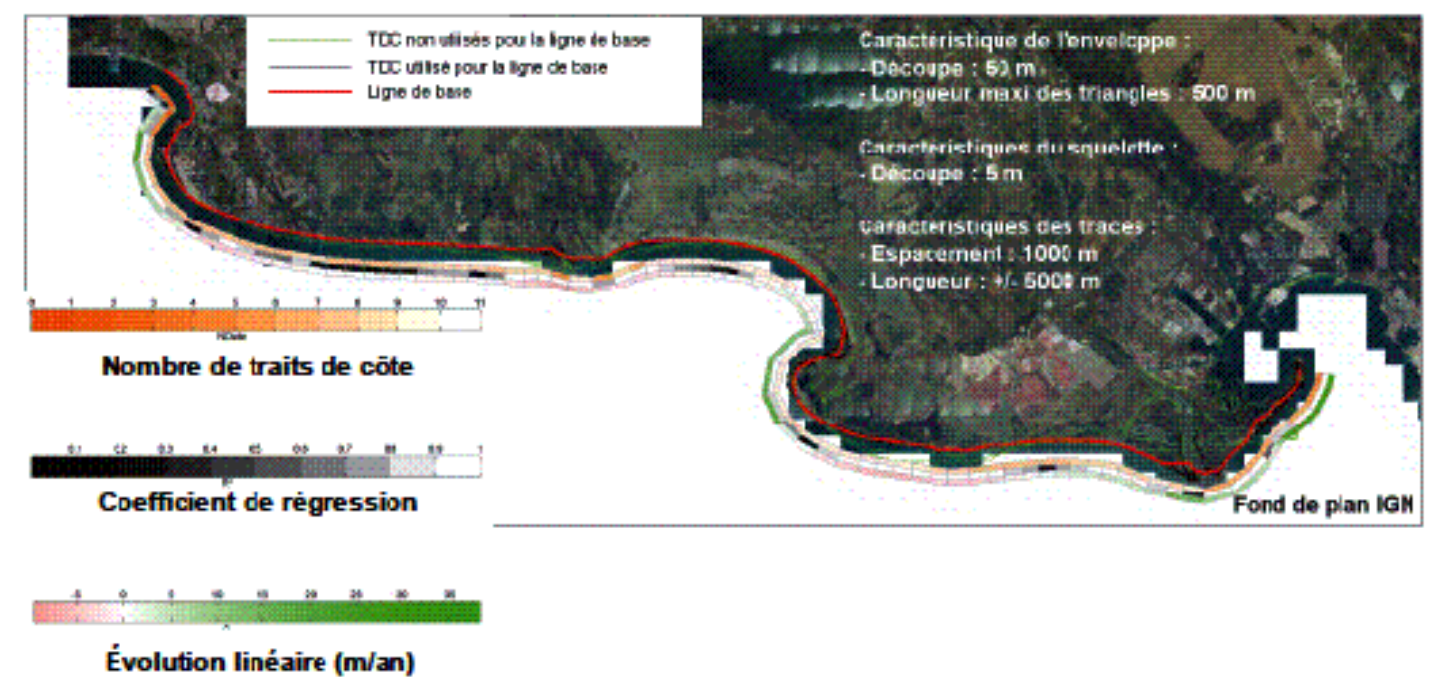

Figure 4. Traitement d'une longue étendue sableuse par MobiTC.

\subsection{Test 3 : Une flèche sableuse}

Le dernier secteur test de cet article est la flèche sableuse de la Gracieuse (figure 5). Quinze traits de côte ont été utilisés ici, ils datent de 1940 à 2003. Ce test a nécessité un développement spécial pour traiter les flèches sableuses. Pour l'élaboration de la ligne de base, l'enveloppe s'appuie sur les traits de côte qui dans la plupart des cas (et c'est ce que les auteurs recommandent lors de l'acquisition de trait de côte dans le cas de flèche) font le tour de la flèche. Ainsi l'enveloppe correspond à l'évolution totale de la flèche et la ligne de base représente bien la forme "moyenne" de la flèche.

Dans le cas de flèche, la ligne de base est parcourue d'abord dans un sens pour calculer l'évolution côté mer, puis à l'extrémité quelques traces sont générées pour l'évolution du bout et ensuite la ligne de base est parcourue en sens inverse pour l'évolution côté terre. 


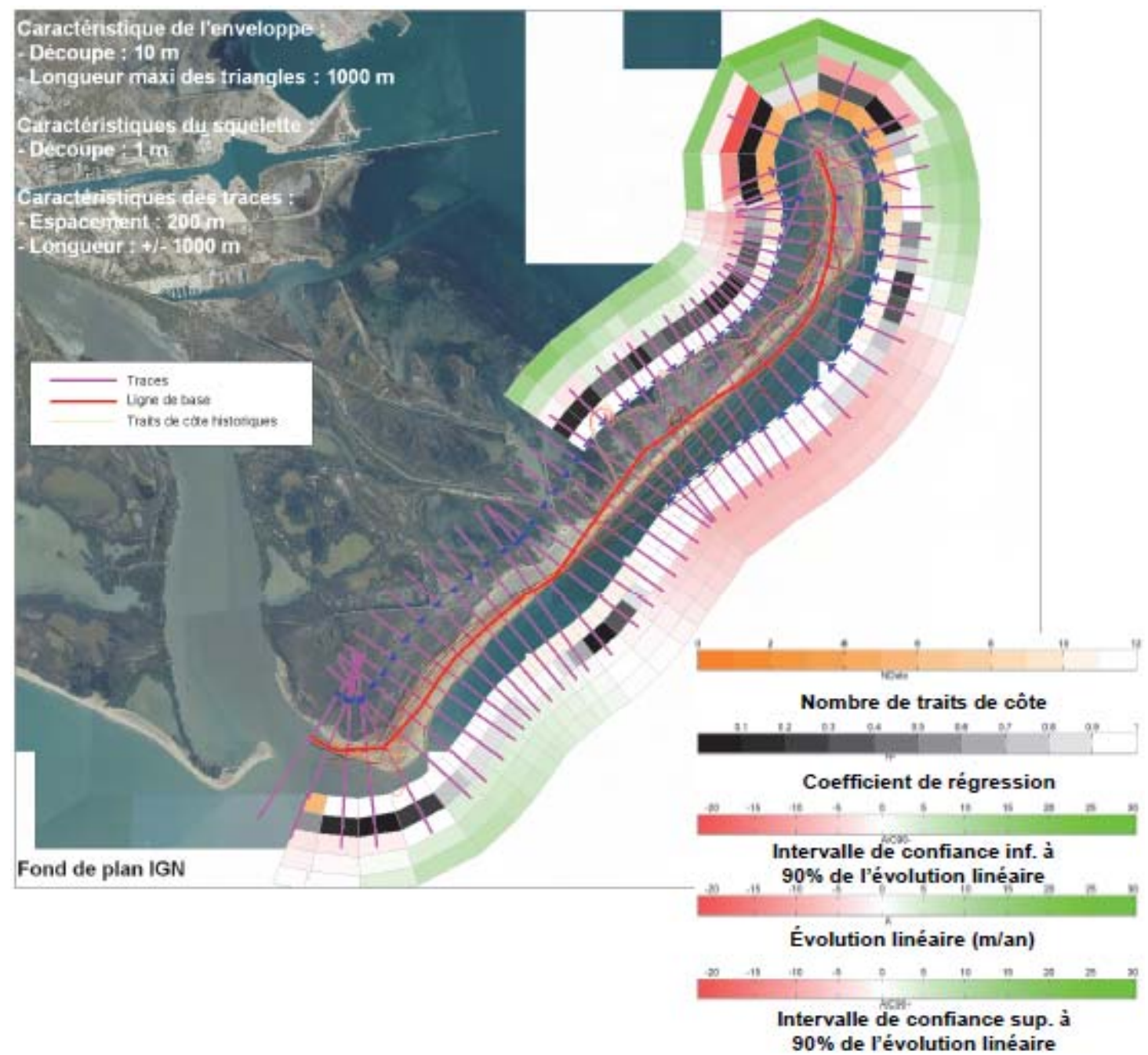

Figure 5. Traitement d'une flèche sableuse par MobiTC.

\section{Conclusion et perspectives}

L'outil MobiTC offre la possibilité d'étudier la mobilité des traits de côte historiques sur des territoires à forme complexe grâce à sa génération automatique de la ligne de base, avec un souci de simplicité pour l'utilisateur. De nombreux indicateurs sont calculés permettant à l'utilisateur de mener une analyse critique de la mobilité.

MobiTC procure également un moyen de capitaliser des traits de côte avec leurs métadonnées de façon rigoureuse.

Il est projeté d'utiliser MobiTC dans le cadre de l'actualisation des catalogues sédimentologiques pour déterminer des vitesses d'avancée et de recul à l'échelle nationale. Cela sera réalisé à partir de traits de côte digitalisés sur orthophotographies à l'échelle de la France mais aussi de traits de côte qui seront mis à disposition. Plus largement MobiTC sera utilisé dans le cadre de la stratégie nationale de gestion du trait de côte pour à terme déterminer des territoires à risque érosion (TRE). 


\section{XII ${ }^{\text {èmes }}$ Journées Nationales Génie Côtier - Génie Civil \\ Cherbourg, 12-14 juin 2012}

\section{Remerciements}

Le développement de MobiTC a été réalisé dans le cadre de l'actualisation des catalogues sédimentologiques piloté par le CETMEF pour le Ministère de l'Ecologie et en particulier la Direction Générale de l'Aménagement, du Logement et de la Nature et la Direction Générale de la Prévention des Risques.

\section{Références bibliographiques}

CROWELL M., LEATHERMAN S.P., BUCKLEY M.K. (1991). Historical shoreline change: error analysis and mapping accuracy. Journal of Coastal Research, Vol. 7, $\mathrm{n}^{\circ} 3$, pp 839-852.

FENSTER M., DOLAN R. (1994). Large-scale reversals in shoreline trends along the U.S. mid-Atlantic coast. Geology 22, nº 6 (Juin), pp 543-546.

doi:10.1130/0091-7613(1994)022<0543:LSRIST>2.3.CO;2

GENZ A.S., FLETCHER C.H., DUNN R.A., FRAZER L.N., ROONEY J.J. (2007). The predictive accuracy of shoreline change rate methods and alongshore beach variation on Maui, Hawaii. Journal of Coastal Research, Vol. 23, n 1, pp 87-105.

doi:10.2112/05-0521.1

HIMMELSTOSS E. (2009). DSAS 4.0 Installation Instructions and User Guide. Digital Shoreline Analysis System (DSAS) version 4.0 - An ArcGIS extension for calculating shoreline change, U.S. Geological Survey open-File Report 2008-1278, Thieler, E.R., Himmelstoss, E.A., Zichichi, J.L.

PONS F. (2010). Hydraulic study of the marseille vieux-port river basin. ISTE LTD and John Wiley \& Sons, Inc Environmental hydraulics, Pratical Applications in Engineering edited by Jean-Michel Tanguy, pp 167-181.

WALTON JR. T.L. (1999). Shoreline rythmic pattern analysis. Journal of Coastal Research. Vol. 15, n² 2, pp 379-387. 
Thème 2 - Dynamique sédimentaire 